\title{
IMAGE VELOCIMETRY AND STATISTICAL ANALYSIS OF A MESH-COUPLED AXIAL BLADE DISTRIBUTOR FOR MASS TRANSFER IN A SWIRLING BED
}

\author{
Muhammad Asif Javed, Shazia Shukrullah, Muhammad Yasin Naz, \\ RAJA AdIL SARFRAZ \\ University of Agriculture Faisalabad, Department of Physics, Pakistan \\ e-mail: yasin603@yahoo.com
}

\begin{abstract}
A mesh-coupled axial blade distributor was tested for fluidization of particles in a swirling fluidized bed. The bed velocity was estimated experimentally using a high-speed imaging device and MATLAB supported particle image velocimetry (PIV). The bed velocity was also predicted statistically with a response surface analysis method. During statistical analysis, the confidence interval of bed velocity remained between 0.49485 and 0.49998 . The bed velocity was measured about $0.49741 \mathrm{~m} / \mathrm{s}$ and $0.538 \mathrm{~m} / \mathrm{s}$ through experimental and statistical methods, respectively. The experimental and statistical analysis revealed similar bed weights and superficial velocities with a slight difference of $6.4^{\circ}$ in blade angles.
\end{abstract}

Keywords: mesh-coupled distributor, blade inclination angle, particle image velocimetry, response surface analysis

\section{Introduction}

Fluidization is a process for converting static-solid materials in a settled bed into fluid-like liquid materials by a bottom-up gas or air flow (Yang, 2003). The upward flowing air enters from the plenum into the bed column through a distributor plate. The bed material to be fluidized is placed on the distributor plate. The upstream fluid exerts a pressure or force on the material, known as the drag force, which transforms the static state of the bed into a dynamic state. The net force acting on the bed is a total of the drag force acting upward and downward weight of the bed. The net force, acting downward, should be zero in order to initiate fluidization. The upward drag force increases gradually with a rise in the upward air flowrate. Once the weight of the bed material is equal to the drag force, it cancels the effective bed weight (Sreenivasan et al., 2002; Sulaiman et al., 2016). At this stage, arrangement of the bed material starts to disturb. The bed material starts following all degrees of freedom and said to be fluidized. The characteristics of such gas-solid interaction are very valuable for fast solid-air mixing, uniform temperature distribution and mass and heat transfer. Many chemical and mechanical processes of enhanced efficiency are possible through utilization of the fluidization phenomenon (Wu and Zhan, 2007; Gupta and Sathiamoorthy, 1999). Fluidized bed processing shows many advantages over conventional methods, like high process efficiency, flexible reaction assembly, uniform heat distribution, ability to add or remove materials continuously, uniform mixing of materials, ability to adapt higher temperatures and pressures (Gupta and Sathiamoorthy, 1999).

Several designs, known as conventional fluidized beds, are a result of efforts to enhance working performance of fluidized beds. The known conventional beds are centrifugal fluidized beds, circulating fluidized beds, vibro-fluidized beds, tapered fluidized beds, spouted fluidized beds, etc. (Amornsirirat et al., 2011). The fluidized beds are being used in combustion, gasification, drying, heating and catalytic cracking, surface treatment, surface coating, gas separation, mass 
transfer, etc. (Rao et al., 2007; Jangam et al., 2009; Kulkarni and Joshi, 2011). However, presence of moving parts, higher pressure drops across the distributor plate, insufficient gas-solid interactions, inappropriate mixing of bed materials, poor processing of different shapes and sizes and complex hydrodynamics are the major drawbacks of the conventional fluidized beds. Swirling Fluidized Bed (SFB) is relatively a newer version, which is developed to mitigate the drawbacks of the conventional fluidized beds. In this design, the air enters the bed column from the plenum chamber via an air distributor. The distributor design is made to add swirling motion to the air flow. This is the hallmark of a SFB compared to the conventional fluidized bed designs (Sutar and Sahoo, 2011). The air distributor design considerably impacts the quality of fluidization and overall process cost. As the swirl motion is imparted to the gas flow due to inclined air intake, the pressure drop required to fluidize the bed material is generally lower compared to the conventional models. The power, required for SFB processing of materials, is therefore also lower than in the conventional models.

The most important part of the fluidized beds is the air distribution system which is supposed to distribute the air evenly throughout the bed and to support the material in the settled bed (Paiva et al., 2009). The efficiency of operation depends upon design of the air distributor. Various air distributor designs were reported in the past (Sutar and Sahoo, 2011; Paiva et al., 2009; Brink et al., 2011; Faizal et al., 2012). The most popular air distributor geometry for SFBs is the annular blade distributor. This concept was first presented by Ouyang and Levenspiel (1986). The distributor design was further improved by Shu et al. (2000). Sutar and Sahoo (2011) tested three geometries of an annular blade distributor, namely inclined holes distributor, single row and three rows vane type distributors. The idea of a helical nozzle to provide swirling motion in the fluidizing medium was presented by Aworinde et al. (2015). The effect of different parameters of the annular blade distributor, such as inclination angle of blades and properties of bed particles was studied by Naz et al. (2016). Although a reasonable amount of literature has been reported on SFBs, the issues of high pressure drop and selection on size of the bed material remain unresolved. High pressure drop requires additional pumping power and increases the cost of the process. Limitations on material size reduce SFBs performance and applicability.

The above discussed issues can be addressed by deliberately modifying the existing designs of annular blade distributors. In the given work, a hybrid model of the distributor is proposed by coupling an annular blade distributor with a wire mesh. The wire mesh was placed on the top of the annular distributor. The mesh coupling at the top of the annular distributor resulted in a hybrid model that was ideal for processing a variety of particle sizes without impacting the pressure drop too much. This air distributor model blends characteristics of the mesh type air distributor with the annular blade distributor. The mesh at the top stops the fall back of the bed material into the plenum chamber. The particles falling back can stay in the gaps between the blades and increase the pressure drop across the air distribution system. The objective of this work was to study the effect of blade inclination angle, bed weight and superficial velocity on bed hydrodynamics by considering a mesh-coupled blade distributor.

\section{Experimental setup}

The presented setup consisted of a fluidized bed, domestically designed mesh-coupled air distributor, high speed imaging device and MATLAB supported Particle Image Velocimetry (PIV) software. The diameter and length of the cylindrical iron bed column were $30 \mathrm{~cm}$ and $50 \mathrm{~cm}$, respectively, as shown in Fig. 1. The air distributor, which is the most important part of the fluidized bed, was fabricated by coupling a mesh of pore size $2.8 \times 2.8 \mathrm{~mm}^{2}$ to the annular blade distributor. The blade inclination angles were fixed at $30^{\circ}, 45^{\circ}, 60^{\circ}, 75^{\circ}$ and $90^{\circ}$, as illustrated in Fig. 2. The central body in the form of a cylinder was placed in the middle of the bed column to avoid formation of dead zones and clogging. 


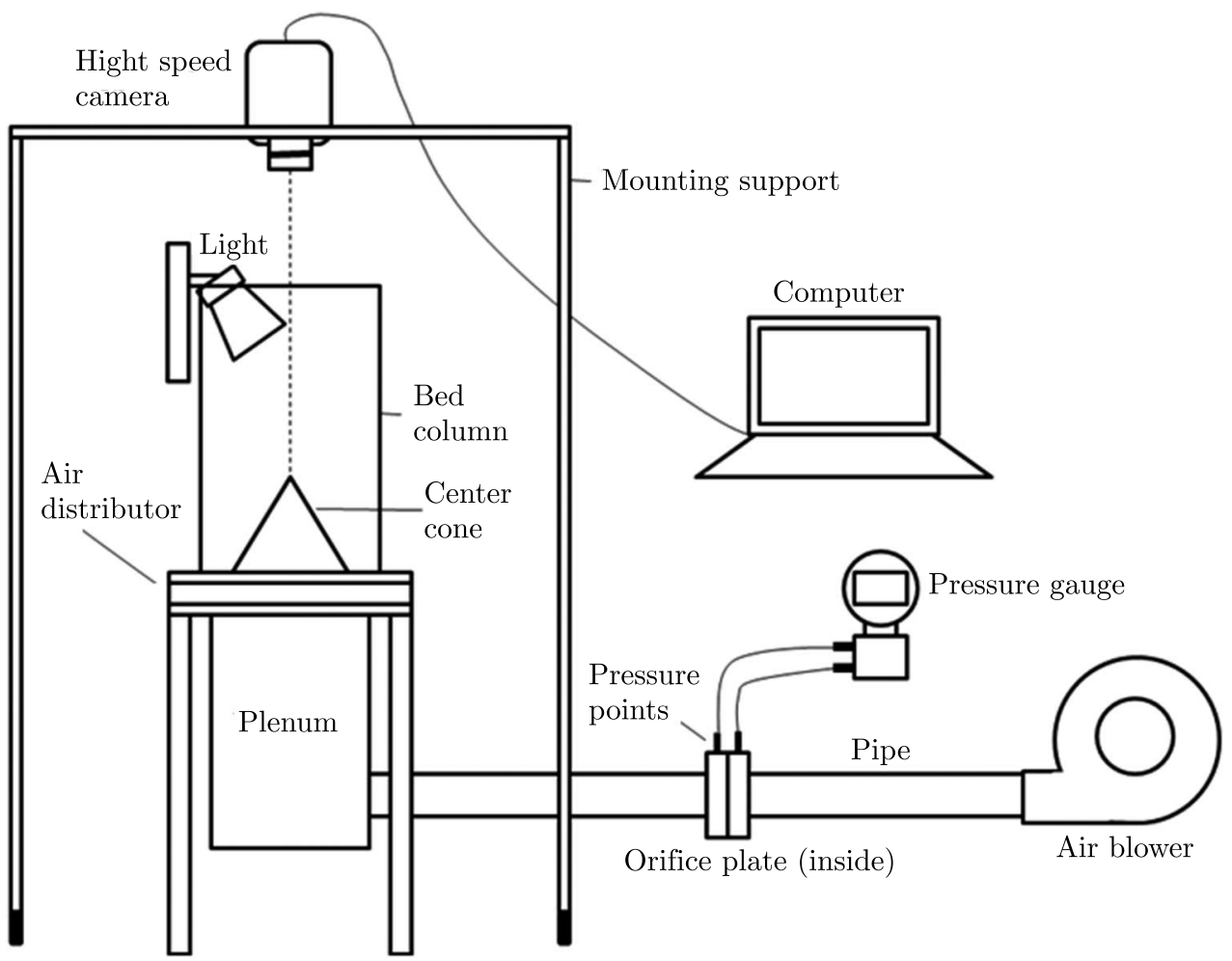

Fig. 1. Schematic of a complete SFB experimental setup

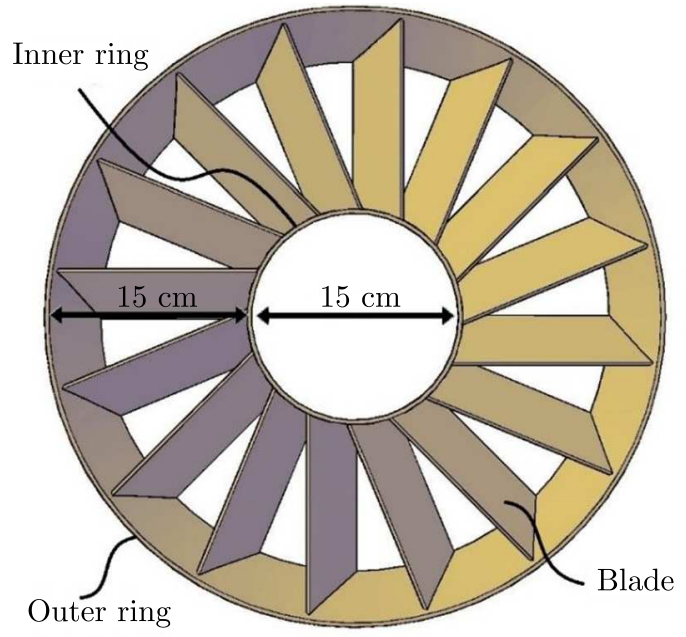

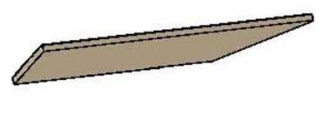

$30^{\circ}$

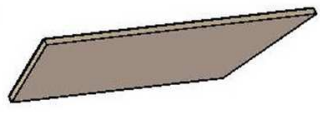

$45^{\circ}$

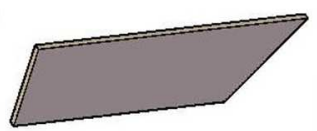

$60^{\circ}$

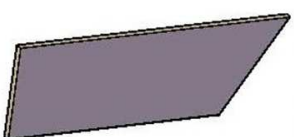

$75^{\circ}$

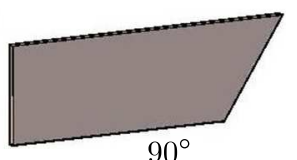

$90^{\circ}$

Fig. 2. Annular blade distributor and orientation of blades at different inclination angles

Figure 3 shows a complete design of the annular blade distributor coupled with the wire mesh. The diameter of inner and outer rings of the distributor are $15 \mathrm{~cm}$ and $30 \mathrm{~cm}$, respectively. The diameter of the outer ring exactly matches the diameter of the bed column and plenum chamber. A metallic collar was welded around the neck of the plenum chamber to hold the air distributor between the bed column and chamber. A blower of $8 \mathrm{~kW}$ was used to pump the air 
into the plenum chamber at different flowrates. The corresponding pressure drop was measured by placing an orifice plate in the flowline. A manometer was connected to the orifice plate for pressure drop measurements. These measurements were used to estimate the superficial air velocity required to fluidize a settled bed.

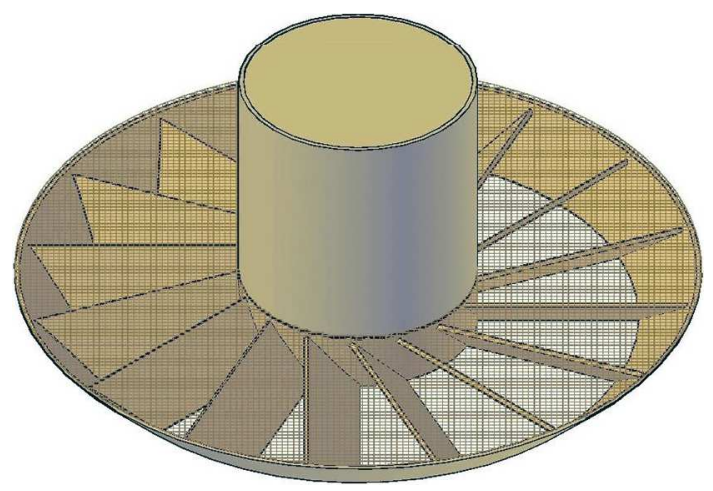

Fig. 3. Complete design of an air distributor having a central solid cylinder. The blade assembly is covered with a wire mesh

The settled bed was fluidized by blowing the bed material with a blower. The swirling motion to the bed was imparted by the inclined entry of the air into the plenum chamber by a tapered pipe and inclined blades of the air distributor. The settled bed started to readjust with a steady increase in the air flow. The moment when fluidization started, the bed entered incipient fluidization stage and the corresponding superficial air velocity was named as the minimum air velocity for fluidization. With a further rise in the air flow, the bed entered the bubbling regime and, finally, the uniform swirling regime of fluidization. The bed dynamics were elaborated in this regime of uniform fluidization. PIV technique was used to find out the flow field of particles, trajectories and velocities. The spherical plastic beads were used as a bed material in this work. White and black particles were mixed in 1:3 ratio. The white particles were used as tracer particles as they better reflect the light than the dark particles. The black particles formed a dark background for white particles during high speed imaging. The size and density of the particles were $6 \mathrm{~mm}$ and $1.772 \mathrm{~g} / \mathrm{cm}^{3}$, respectively.

The swirling bed was made visible by a halogen bulb to facilitate photographing of the bed, using a high-speed camera. The camera was operated at a framerate of $1000 \mathrm{fps}$ and captured images were stored in a memory device for further process with PIV software. At this framerate, the camera resolution was $856 \times 846$. In PIV analysis, the calibration was done by considering dimensions of the bed column and the central cylinder. As fluidization was uniform throughout the bed, only a quarter of the bed was selected for PIV analysis. The rest of the bed area was masked during pre-PIV processing of the images. The velocity vector field of particles in the swirling bed was generated by comparing the interrogation windows in consecutive images. From the vector field, the velocity magnitude was obtained as a function of the blade inclination angle, bed weight and superficial air velocity.

\section{Results and discussions}

During PIV investigations, the bed particles were tracked by finding the highest similarity between the flow patterns in consecutive images. A small portion of images was selected for such comparison, known as the interrogation window. The size of the interrogation window for the present study was selected to be $32 \times 32$ pixels. The region that does not provide any valuable fluidization data has been deleted. The correlation between the questioning windows of consecutive images, taken after a specific time interval, provided the distance covered by the tracer particles. 
The velocity of the particles was obtained by dividing the distance with the time interval. Three types of filters, i.e. signal to noise ratio filtering, local filtering and global filtering were employed to remove the unwanted velocity vectors. The removal of such unwanted velocity vectors created empty spaces, which were filled up by interpolation of the velocity vectors from nearby regions. The average velocity magnitude at different points on the vector field was obtained by averaging five velocity measurements.

Figure 4 shows a typical vector field for bed weight of $500 \mathrm{~g}$, blade angle of $60^{\circ}$ and superficial velocity of $2.31 \mathrm{~m} / \mathrm{s}$. The particle size and density were kept constant to be $6 \mathrm{~mm}$ and $971.43 \mathrm{~kg} / \mathrm{m}^{3}$, respectively. The bed velocity was non-uniform, i.e. yellow color in the vector field corresponds to the highest velocity, whereas blue color shows the lowest velocity. The velocity was lower near the bed edges, i.e. close to the wall and central cylinder. The frictional forces were the source of reduction in particle velocity (Sulaiman et al., 2016).

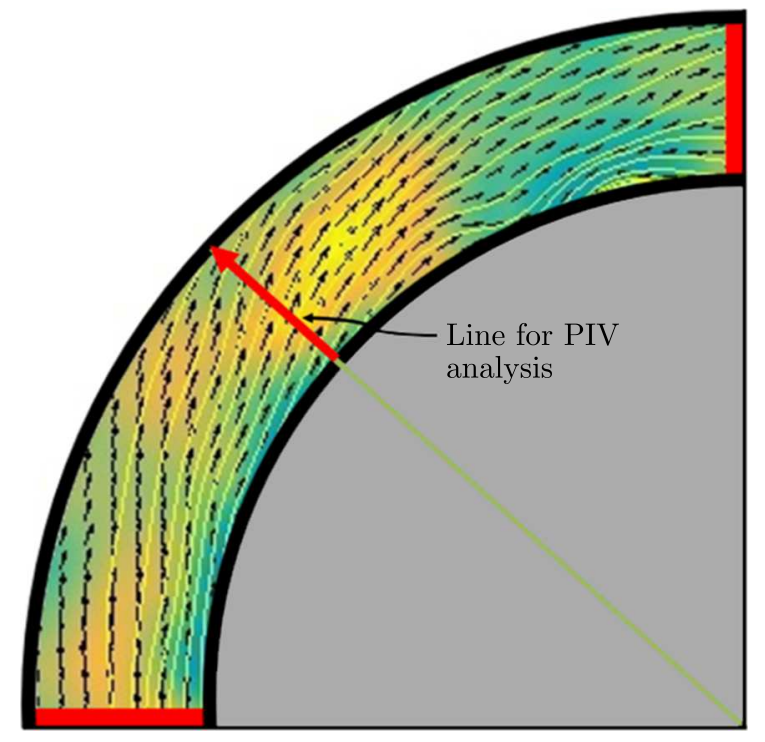

Fig. 4. A velocity vector field of $500 \mathrm{~g}$ of bed material

Due to higher momentum transfer, the bed velocity, as calculated by PIV software, was increased with an increase in superficial air velocity. The velocity was highest in the middle of the bed and decreased slowly toward the bed boundaries. As the air velocity rose, there was no change in bed velocity near the bed column wall due to an increase in the centrifugal force with rising air velocity. Figure 5 reveals the trend of bed velocity with distance on the line between the central cylinder and the bed column wall. The line for bed velocity is drawn in Fig. 4. For these measurements, the blade angle was $30^{\circ}$ and bed weight was $500 \mathrm{~g}$. The air velocity was increased from $1.52 \mathrm{~m} / \mathrm{s}$ to $2.51 \mathrm{~m} / \mathrm{s}$ with a step size of $0.1 \mathrm{~m} / \mathrm{s}$. For all superficial velocities, the bed velocity demonstrated a supra-linear trend over the distance on the line between the boundaries.

Bed velocity in the middle of the line was noted to be larger but slowly decreased as the measurement point moved to the ends of the line, especially at lower air velocities. This decreasing trend in bed velocity is attributed to frictional forces on the bed material at both ends of the line, particularly at the outer end (Brink et al., 2011). When the particle density is high and the air velocity is below the optimum value, these frictional effects become more detrimental for the bed dynamics. On comparison of the optimized values of air velocity at different blade angles, the highest bed velocity was achieved at an angle of $60^{\circ}$. For this blade angle, the frictional effects on bed dynamics were more pronounced near the bed column wall as compared to the central cylinder (Shukrie et al., 2016). The centrifugal force led the tracer particles to follow the tangential paths. In the response, friction between the particles themselves and between 


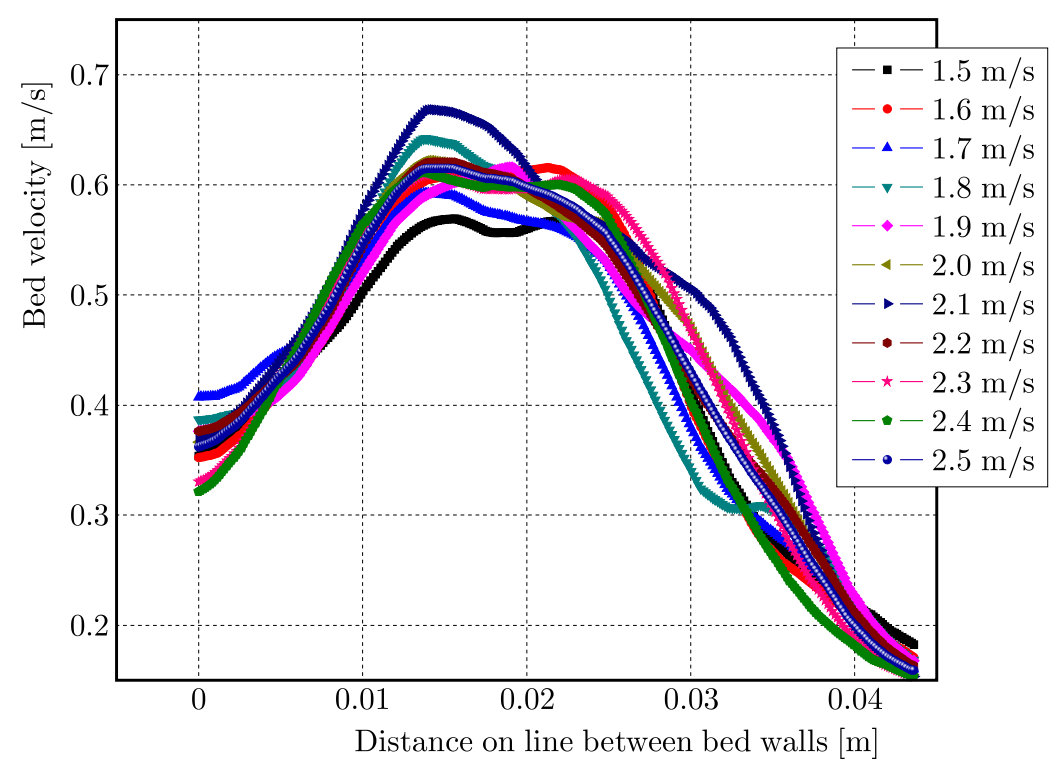

Fig. 5. Bed velocity plots at different air velocities for fixed bed weight of $500 \mathrm{~g}$ and blade angle of $30^{\circ}$

the particles and wall was increased. The peak bed velocity was measured about $0.497 \mathrm{~m} / \mathrm{s}$. Relatively smooth swirling of the bed material happened at air velocity of $2.3 \mathrm{~m} / \mathrm{s}$ regardless of blade angles. Therefore, this air velocity was assumed as the optimum condition for the operation of SBFs having the mesh-coupled annular distributor. These findings are in good agreement with the work of Naz et al. (2015) who also used a hybrid model of the air distributor for SFB operation.

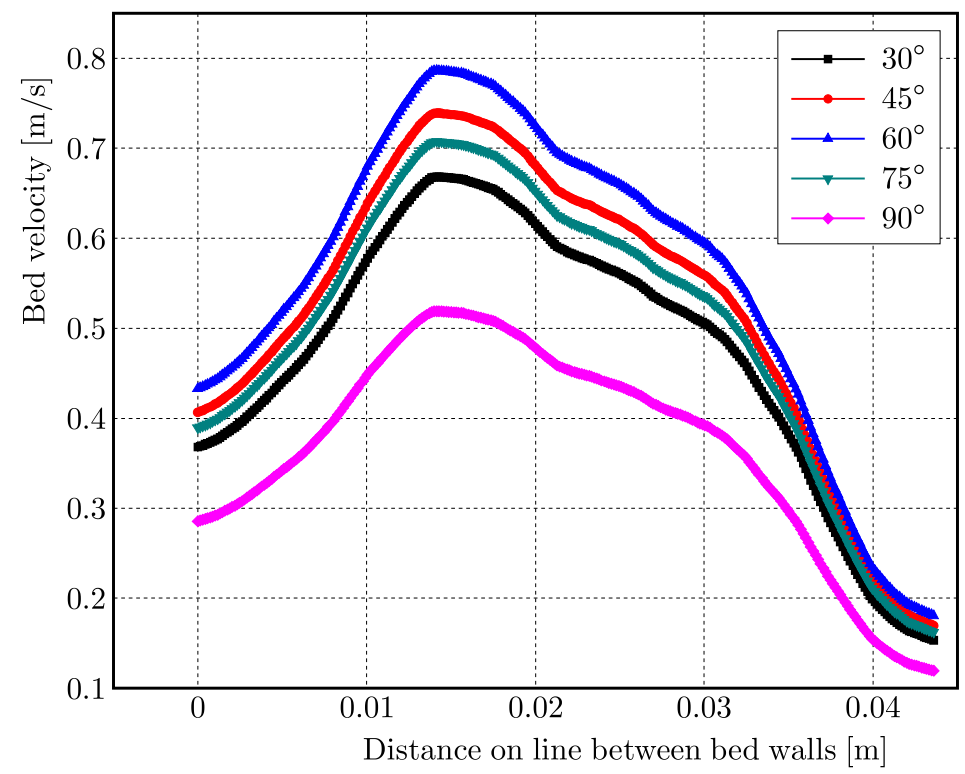

Fig. 6. Trend of bed velocity at different blade angles for fixed bed weight of $500 \mathrm{~g}$ and air velocity of $2.31 \mathrm{~m} / \mathrm{s}$

Figure 6 reports the bed velocity for different fixed blade angles. The air velocity and bed weights were fixed at $2.3 \mathrm{~m} / \mathrm{s}$ and $500 \mathrm{~g}$, respectively. The highest bed velocity and uniform fluidization operation were possible at a blade angle of $60^{\circ}$. The possible reason for $60^{\circ}$ to be the optimized blade angle is that the magnitudes of horizontal and vertical components of the air velocity are not equal at this angle. The vertical component is a bit large as compared to 
the horizontal component. This combination of the horizontal and vertical velocity components causes uniform fluidization and large swirling velocity. The fluidization was somewhat turbulent in the beginning, which became smooth with a rise of the air flow. The air flow was increased to suitable fluidization conditions by minimizing bubbling of the bed. The results of past studies, conducted by Joseph Kunju (Shukrie et al., 2016) and Mohideen et al. (2012), are in good agreement with the presented data. However, they used simple annular blade distributors rather than hybrid models.

The trend of pressure drop with air velocity is shown in Fig. 7. The plots were obtained for different fixed blade angles. The pressure drop across the distributor was noted to increase as the air velocity gradually increased. Conversely, the pressure drop decreased as the blade angle increased. A blade angle of $60^{\circ}$ was found to be the optimal angle to achieve uniform and balanced fluidization. For this blade angle, the pressure drop through the distributor was mild. A high pressure drop is considered undesirable as it requires more power to satisfy the condition of complete fluidization (Rees et al., 2006).

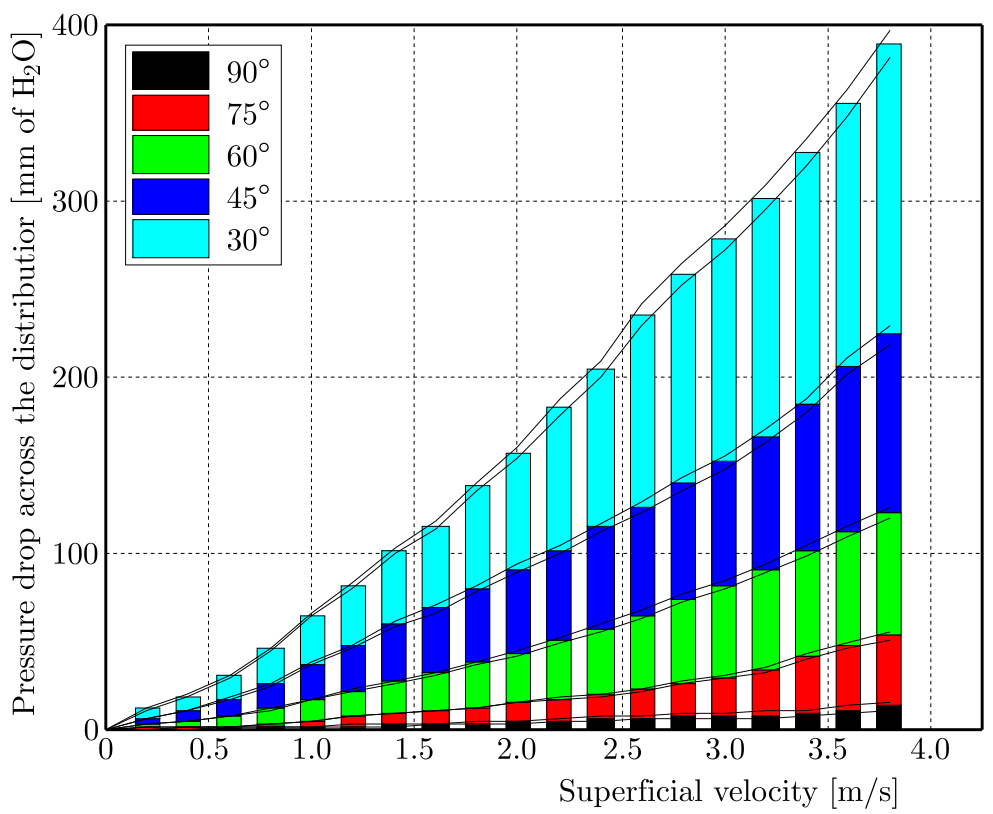

Fig. 7. Effect of air velocity on pressure drop at different blade angles

Figure 8 shows the plot of bed pressure drop versus superficial air velocity, when the blade inclination angle and bed weight were $60^{\circ}$ and $500 \mathrm{~g}$. Many practical correlations are available for predicting the minimum fluidization velocity $U_{m f}$ in conventional beds, but no correlation exists for $U_{m f}$ in SFBs. Therefore, $U_{m f}$ is determined experimentally for SFBs (Milovanov et al., 2015). The plot in Fig. 8 was used to find $U_{m f}$, which corresponds to the air velocity at the point where the two straight lines on the plot intersect each other. With a positive change in air velocity in the settled bed, the pressure drop increases linearly during the early stages of fluidization. With a further increase in air velocity, the pressure drop starts gaining constant values. From the pressure drop plot, $U_{m f}$ is estimated to be about $1.6 \mathrm{~m} / \mathrm{s}$. This is the minimum velocity of the bottom-up air flow required to initiate incipient fluidization. All the fluidized beds operated at air velocities between the terminal and minimum fluidization velocity. The ratio of these velocities defines the range of bed operation. The ratio of the superficial air velocity $U_{\text {sup }}$ and minimum fluidization velocity $U_{m f}$ is used to design fluidized bed reactors.

The effect of blade angle on $U_{m f}$ is reported in Fig. 9. The blade angle was varied from $30^{\circ}$ to $90^{\circ}$ to study its impact on quality of fluidization. It is evident from the plot that with an increase in blade angle, $U_{m f}$ decreases slightly. The reason for this decrease are large open spaces 


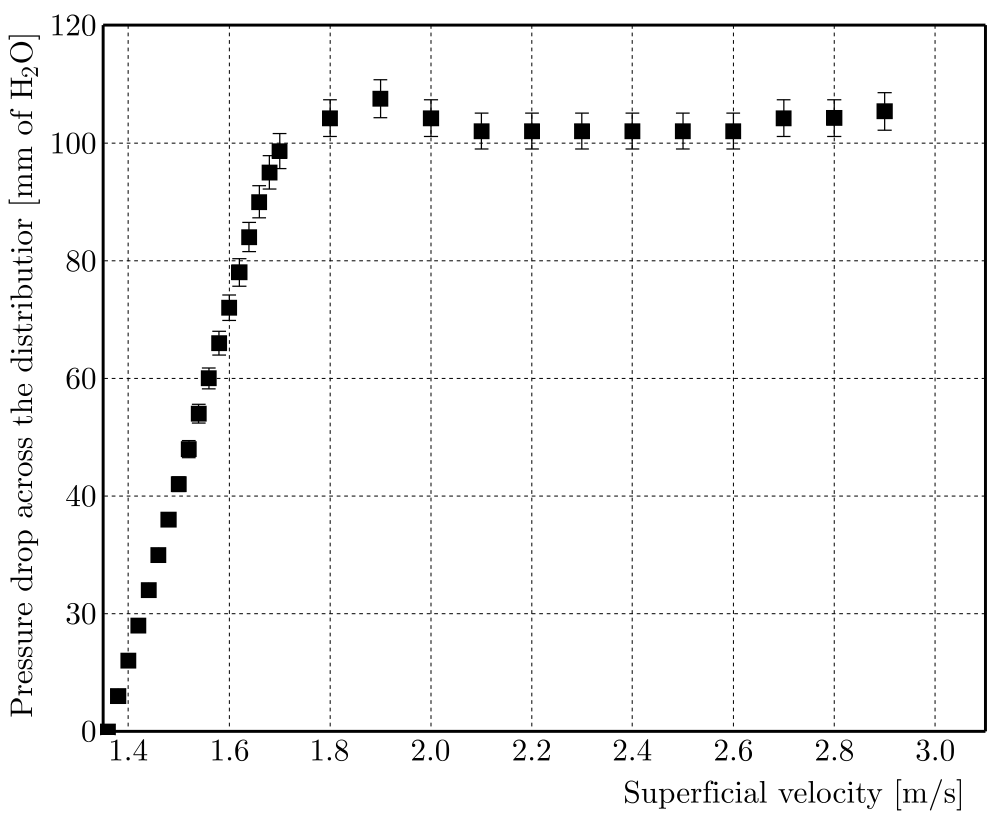

Fig. 8. Trend of pressure drop with superficial velocity for estimation of the minimum fluidization velocity

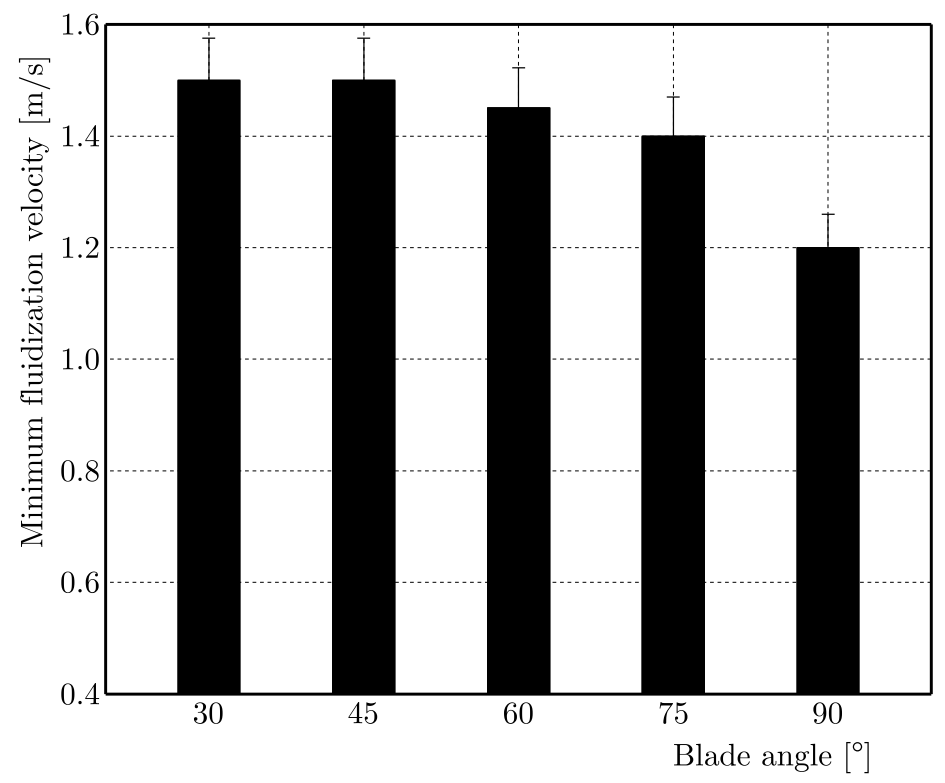

Fig. 9. Change in the minimum fluidization velocity with blade angle

between distributor blades, which facilitate an easy flow of the air and make fluidization an easy task. An increase in blade angle above the optimal value, however, deteriorates the fluidization quality (Kumar and Murthy, 2010). Below the optimal angle, the effective surface area of blades is large enough to impede the air flow through the distributor into the bed. The air bounces back and develops back pressure in the flowline. However, Venkiteswaran et al. (2015) reported different findings on the effect of blade angle on $U_{m f}$. In their study, no notable change $U_{m f}$ was noticed with an increase in blade angle from $10^{\circ}$ to $20^{\circ}$. They investigated only three blade angles $\left(10^{\circ}, 15^{\circ}\right.$ and $\left.20^{\circ}\right)$ with a small step size of $5^{\circ}$. The difference in percentage of effective surface areas for this step size was not large enough to effect $U_{m f}$. In this work, five blade angles were tested with a relatively bigger step size of $15^{\circ}$. A noticeable difference in percentage of effective surface areas was noticed for this step size, which might have effected $U_{m f}$ at higher blade angles (Miin et al., 2015). 
The settled bed to fluidized bed height is reported in Fig. 10. The height of the bed in an unfluidized state is known as the static bed height. The bed height depends upon the bed weight particle shape and size. The fluidized bed height is always higher than the settled bed height. The particles in a fluidized bed detach from each other by increasing the interstitial sites area (Naz et al., 2015). In the fluidized state, SFB is free of bubbles like a liquid-solid fluidized bed and displays stable fluidized bed behavior. Figure 10 shows the trend of bed height with change in superficial velocity for different blade angles. The bed height increases with an increase in blade angle. Larger blade angles proved to give more open areas for the air flow through the distributor. For smaller blade angles, the swirling motion of bed particles becomes vigorous, resulting in a large centrifugal force and hence large downward frictional effects which detain the bed expansion. It is worth mentioning here that the bed expansion starts only after the air velocity crosses the minimum fluidization velocity.

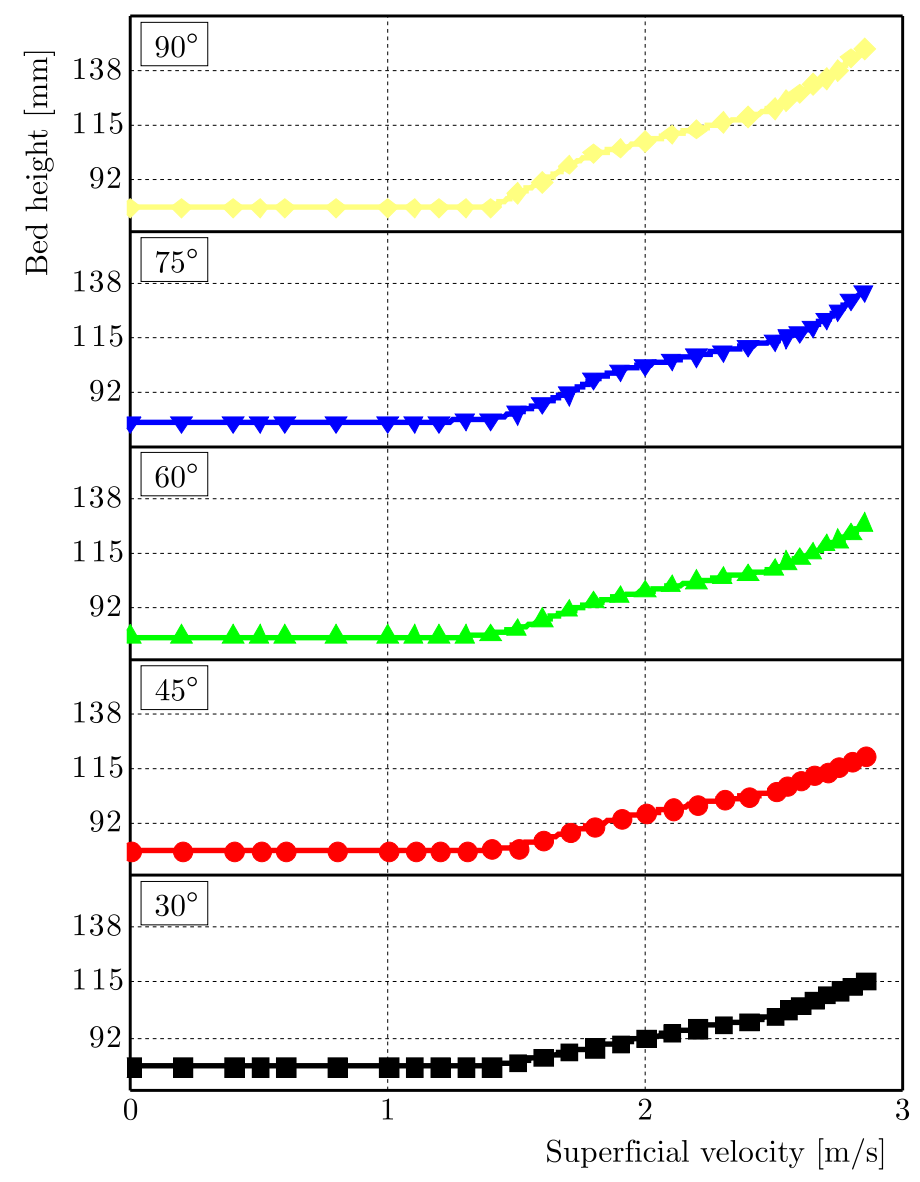

Fig. 10. Change in bed height at different blade angles

Figure 11 demonstrates a change in bed heights with superficial velocity at different bed weights. It is evident from the plot that as the bed weight increases, the bed height also increases. Increasing the weight of the bed means increasing the number of particles that need more space in the bed column (Padhi et al., 2016).

\section{Statistical analysis of bed operation}

The data obtained was in large numbers and a plethora of information, making it difficult to decide about the more and less important factors as well as the optimized values of factors to maximize the output. Response surface regression analysis was used to analyze the data, using 


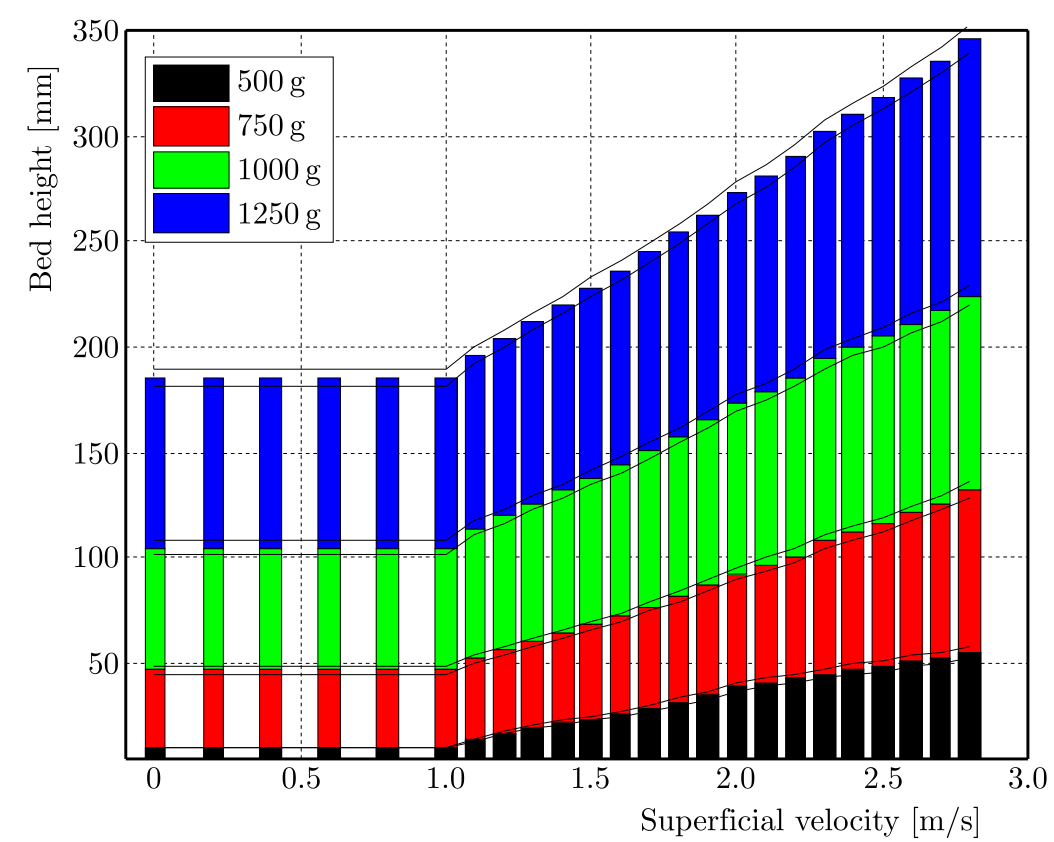

Fig. 11. Change in bed height with superficial velocity for different bed weights

NCSS statistical software. In the statistical analysis, the optimized values of input variables and the maximized output values were obtained, as shown in Table 1. They show that the response variable or bed velocity fitted value is close enough to the desirable value, evidencing the efficiency of the system. Table 1 shows the optimized values of factors which are $500 \mathrm{~g}$ bed weight, $53.6^{\circ}$ blade inclination angle which is nearest to the $60^{\circ}$, and superficial velocity of $2.3 \mathrm{~m} / \mathrm{s}$. The fit confidence interval and probability interval are given in Table 2 .

Table 1. Solution of statistical analysis

\begin{tabular}{|c|c|c|c|c|c|}
\hline Solution & Bed weight & Bed angle & $\begin{array}{c}\text { Air } \\
\text { velocity }\end{array}$ & $\begin{array}{c}\text { Bed } \\
\text { velocity fit }\end{array}$ & $\begin{array}{c}\text { Composite } \\
\text { desirability }\end{array}$ \\
\hline \hline 1 & 500 & 53.6364 & 2.34848 & 0.497413 & 0.623459 \\
\hline
\end{tabular}

Table 2. Fit, confidence interval and probability interval

\begin{tabular}{|c|c|c|c|c|}
\hline Response & Fit & $\begin{array}{c}\text { Standard } \\
\text { error }\end{array}$ & $\begin{array}{c}95 \% \text { confidence } \\
\text { interval }\end{array}$ & $\begin{array}{c}95 \% \text { probability } \\
\text { interval }\end{array}$ \\
\hline \hline Bed velocity (R) & 0.49741 & 0.0013 & $(0.49485,0.49998)$ & $(0.27406,0.72076)$ \\
\hline
\end{tabular}

Figure 12 shows contour plots showing the interaction between the response variable, i.e. bed velocity and the combinations of factors. The first contour plot depicts the combined effect of bed weight and blade inclination angle on bed velocity. The bed velocity is anticipated to be maximum for bed weight of $500 \mathrm{~g}$ and inclination angle of $60^{\circ}$. Similarly, in the second and third contour plot, the combined effect of superficial air velocity and bed weight as well as superficial air velocity and blade inclination angle are depicted. When the superficial air velocity is $2.3 \mathrm{~m} / \mathrm{s}$, the maximum bed velocity is predicted. The color codes depict the bed velocity, dark green to be the minimum and dark blue to be the maximum value. The statistical predictions validated the experimental results as the value of the response variable was 0.4974, close enough to the desired value, i.e. 0.6234 , also the confidence interval was $95 \%$. 
(a)

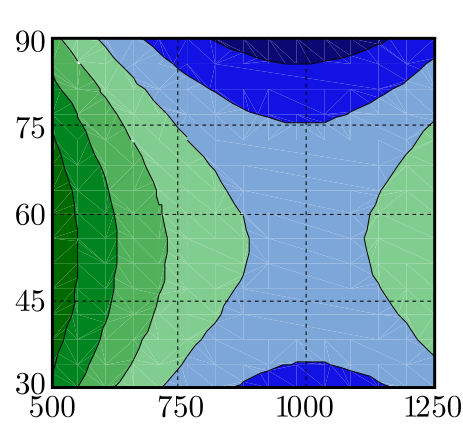

(b)

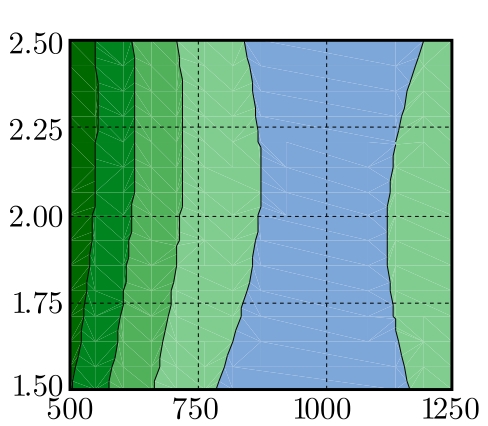

(c)

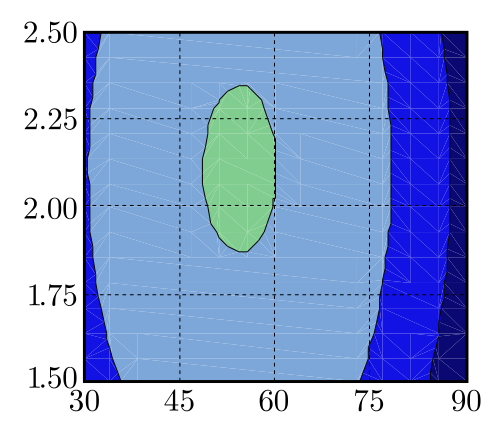

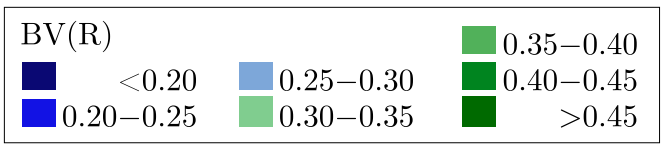

Fig. 12. Contour plots of the response versus factors

\section{Conclusions}

The hydrodynamics of bed particles in uniform swirling regime of operation of SFB was investigated by the particle image velocimetry (PIV) characterization technique. A newly constructed mesh-coupled annular blade distributor was employed in SFB to provide air distribution without shortcomings of the earlier reported distributor versions. The bed velocity was found to be lower for lower superficial air velocity towards the ends of the line joining the central cylinder and boundary of the bed column, on which the bed velocity was determined. The bed velocity was higher at the center of the line. As the superficial air velocity was increased, the bed velocity near the central cylinder was also increased. A polynomial regression model was used to compare the experimental results with the statistical predictions. The bed velocity, which was the response factor, was maximum when bed weight was $500 \mathrm{~g}$. The experimental superficial velocity was found $2.3 \mathrm{~m} / \mathrm{s}$ and blade inclination angle was $60^{\circ}$. The statistically predicted values of bed weight, superficial velocity and blade angle were $500 \mathrm{~g}, 2.348 \mathrm{~m} / \mathrm{s}$ and $54.6^{\circ}$.

\section{Acknowledgements}

Authors would like to thank Mr. Muhammad Arif, Lecturer, Department of Mathematics and Statistics, University of Agriculture Faisalabad for his assistance in performing the statistical analyses.

\section{References}

1. Amornsirirat C.B., Chalermsinsuman B., Mekasut L., Kuchonthara P., Piumsomboon P., 2011, Experiment and 3D simulation of slugging regime in a circulating fluidized bed, Korean Journal of Chemical Engineering, 28, 3, 686-696

2. Aworinde S.M., Holland D.J., Davidson J.F., 2015, Investigation of a swirling flow nozzle for a fluidised bed gas distributor, Chemical Engineering and Science, 2015, 132, 22-31

3. Brink H.G., SaAyman J., Nicol W., 2011, Two dimensional fluidised bed reactor: Performance of a novel multi-vortex distributor, Chemical Engineering Journal, 175, 484-493

4. Faizal M., Seri S., Al-Hafiz M., Raghavan V.R., 2012, CFD studies on velocity distribution of air in a SFB, Advanced Materials Research, 471, 25-29

5. Gupta C.K., Sathiamoorthy D., 1999, Fluid Bed Technology in Materials Processing, CRC Press, Florida, USA

6. Jangam S.V, Mujumdar A.S., Thorat B.N., 2009, Design of an efficient gas distribution system for a fluidized bed dryer, Drying Technology, 27, 11, 1217-1228 
7. Kulkarni A.V., Joshi J.B., 2011, Design and selection of sparger for bubble column reactor. Part II: Optimum sparger type and design, Chemical Engineering Research and Design, 89, 10, 1986-1995

8. Kumar S.H., Murthy D.V.R., 2010, Minimum superficial fluid velocity in a gas-solid swirled fluidized bed, Chemical Engineering Process: Process Intensification, 49, 10, 1095-1100

9. Miin C.S., Sulaiman S.A., Raghavan V.R., Heikal M.R., Naz M.Y., 2015, Hydrodynamics of multi-sized particles in stable regime of a swirling bed, Korean Journal of Chemical Engineering, 32, 11, 2361-2367

10. Milovanov O., Isemin R., Kuzmin S., Mikhalev A., Konyakhin V., Klimov D., 2015, Method for determination of minimum fluidization velocity of polydisperse mixtures in running unit with fluidized bed, International Journal of Energy, 9, 1, 31-37

11. Mohideen M.F., Sreenivasan B., Sulaiman S.A., Raghavan V.R., 2012, Heat transfer in a swirling fluidized bed with Geldart type-D particles, Korean Journal of Chemical Engineering, 29, 7, 862-867

12. Naz M.Y., Sulaiman S.A., 2016, PTV profiling of particles motion from the top and side of a swirling fluidized bed, Journal of Instrumentation, 11, 5, 05019-05036

13. Naz M.Y., Sulaiman S.A., Man Z., Ariwahjoedi B., 2015, Effect of the water spray stress on SFB height, ARPN Journal of Engineering and Applied Sciences, 10, 21, 10192-10195

14. Ouyang F., Levenspiel O., 1986, Spiral distributor for fluidized beds, Industrial Engineering Chemistry Process: Design and Development, 25, 2, 504-507

15. Padhi R.K., Dora D.T.K., Mohanty Y.K., Roy G.K., Sarangi B., 2016, Prediction of bed pressure drop, fluctuation and expansion ratios for three-phase fluidization of ternary mixtures of dolomite in a conical conduit, Cogent Engineering, 3, 1181821-1181840

16. Paiva J.M., Pinho C., Figueiredo F., 2009, Influence of the distributor plate and operating conditions on the fluidization quality of a gas fluidized bed, Chemical Engineering Communications, 196, 342-361

17. Rao K.V.N.S., REDDy G.V., 2007, Effect of distribution design on temperature profiles in fluidized bed during the combustion of rice husk, Combustion Science and Technology, 179, 8, 15891603

18. Rees A.C., Davidson J.F., Dennis J.S., Fennell P.S., Gladden L.F., Hayhurst A.N., Mantle M.D., Müller C.R., Sederman A.J., 2006, The nature of the flow just above the perforated plate distributor of a gas-fluidised bed as imaged using magnetic resonance, Chemical Engineering Science, 61, 6002-6015

19. Shu J., Lakshmanan V.I., Dodson C.E., 2000, Hydrodynamic study of a toroidal fluidized bed reactor, Chemical Engineering and Processing: Process Intensification, 39, 6, 499-506

20. Shukrie A., Anuar S., Oumer A.N., 2016, Air distributor designs for fluidized bed combustors: a review, Engineering, Technology and Applied Science Research, 6, 3, 1029-1034

21. Sreenivasan B., Raghavan V.R., 2002, Hydrodynamics of a swirling fluidised bed, Chemical Engineering and Processing: Process Intensification, 41, 2, 99-106

22. Sulaiman S.A., Minn C.S., Naz M.Y., Raghavan V.R., 2016, Particle Image Velocimetry of a swirling fluidized bed at different blade angles, Chemical Engineering and Technology, 39, 6, 1151-1160

23. Sutar H., Sahoo A., 2011, Effect of distributor-orifice on drying kinetics in a fluidized bed drier, International Journal of Chemical Engineering and Applications, 2, 5, 346-351

24. Venkiteswaran V.K., Ramil H.S. Raghavan V.R., 2015, CFD Simulation of air flow through the annular distributor of a Swirling Fluidized Bed, Applied Mechanics and Materials, 700, 619-625

25. WU C., ZHAN J., 2007, Numerical prediction of particle mixing behavior in a bubbling fluidized bed, Journal of Hydrodynamics, 19, 3, 335-341

26. YANG W.C., EDIT., 2003, Handbook of Fluidization and Fluid-Particle System, CRC Press, New York, USA 